\title{
A study of neurological diseases in farmed deer in Switzerland, with emphasis on chronic wasting disease
}

\author{
Veronika Sieber • Marie-Pierre Ryser-Degiorgis • \\ Catherine Botteron
}

Received: 11 August 2006 /Revised: 28 June 2007 / Accepted: 10 July 2007 / Published online: 3 August 2007

(C) Springer-Verlag 2007

\begin{abstract}
A study of neurological diseases in farmed deer, with emphasis on chronic wasting disease, was conducted during 2 years in Switzerland. Deer breeders were asked to submit the heads of all deer at least 2 years of age, found dead or slaughtered, for examination. A complete histological examination of the brain and immunohistochemical detection of the prion protein on selected regions of the brain and lymphoid tissues were performed on 120 apparently healthy and 40 diseased animals. In a number of cases, a full necropsy was performed. Significant inflammatory and/or degenerative changes were seen in $25 \%$ of the brains. No evidence for a transmissible spongiform encephalopathy was established.
\end{abstract}

Keywords Transmissible spongiform encephalopathy . Survey · Brain · Histopathology · Immunohistochemistry

\section{Introduction}

Chronic wasting disease (CWD) is a transmissible spongiform encephalopathy (TSE) of captive and free-ranging cervids characterised by an accumulation of protease-resistant prion protein $\left(\mathrm{PrP}^{\mathrm{CWD}}\right.$, also known as $\operatorname{PrP}^{\mathrm{SC}}$ or $\left.\operatorname{PrP}^{\mathrm{d}}\right)$ in brain and

Communicated by W. Lutz

V. Sieber · M.-P. Ryser-Degiorgis

Centre for Fish- and Wildlife Health,

Institute of Animal Pathology, Vetsuisse Faculty Berne,

Berne, Switzerland

\section{Botteron}

NeuroCenter, Department of Clinical Veterinary Medicine,

Vetsuisse Faculty Berne,

Berne, Switzerland

\section{Botteron $(\square)$}

Department of Veterinary Pathology,

Faculty of Veterinary Science, University of Liverpool,

Liverpool, UK

e-mail: catherine.botteron@liv.ac.uk lymphoid tissues. Native North American deer (Odocoileus spp.) and elk (Cervus elaphus nelsoni) are known as the natural hosts of CWD. Disease symptoms are non-specific: Loss of body condition and changes in behaviour are the most striking clinical signs. The minimum incubation period is suggested to be 16 to 17 months (Williams and Miller 2002).

CWD was first identified in research facilities in Colorado and Wyoming in 1967 and has been present in free-ranging cervids in these states for more than 20 years. Disease spreading among farmed deer has been attributed to commercial movements of infected but clinically healthy animals (Williams and Miller 2003). Sohn et al. (2002) reported the first case of CWD in Korea in an animal imported from a Canadian farm. So far, CWD has never been recognised in Europe, and targeted investigation in Belgium and Germany did not show any positive cases (Roels et al. 2005; De Bosschere et al. 2006; Schettler et al. 2006). However, as scrapie and bovine spongiform encephalopathy are present on the European continent and considering that a recent study demonstrated the potential transmission of scrapie to elk under experimental conditions (Hamir et al. 2004), the occurrence of CWD in European deer cannot be excluded.

The TSE status of captive deer in Switzerland, as well as the range of other neurological diseases, has never been investigated so far. Therefore, the aim of this study was to examine the Swissfarmed deer population for the presence of neurological diseases, with a special focus on TSE.

\section{Materials and methods}

Sampling

All registered Swiss deer breeders were asked to send material for investigation from February 2003 to January 2005. Requested material consisted in whole carcasses of 
animals found dead or shot for sanitary reasons (diseased animals) and heads of slaughtered healthy deer. Because of the long incubation time of CWD (Williams and Miller 2002), only animals older than 18 months were considered for this study. Participation was on voluntary basis, and the study was anonymous. Transport and examinations were at no cost for the farmers. All deer farmers were asked to shoot animals on the neck to allow for a thorough examination of the brain (as authorised by the Swiss Federal Veterinary Office, Tierversuchsbewilligung No. 53/03). Whenever possible, perished animals were submitted to a full necropsy (Sieber 2006). The heads of slaughtered deer were either collected directly on the farm or received by post. Age, body condition, weight and reason for killing were recorded.

For each animal, the better preserved half of the sagitally cut brain, one palatine tonsil and the parotid, mandibular and lateral retropharyngeal lymph nodes were placed in $10 \%$ buffered formalin. The other half of the brain was kept frozen at $20^{\circ} \mathrm{C}$ for potential further analyses. Multiple representative areas of the brain were sampled: basal nuclei, thalamus, hippocampus, midbrain, neocortex (fronto-temporal and parieto-occipital), cerebellum with pons and medulla oblongata at the level of the obex.

\section{Animals}

One hundred and sixty heads were collected. Because the number of Swissfarmed deer greater than or equal to 18 months old is estimated at 5,200 (Sieber 2006), this sample size corresponds to $3.1 \%$ of the target population. The heads originated from 63 (14.6\%) of the 432 registered deer farms and zoological gardens located in 17 of the 26 Swiss cantons. Between 1 and 14 heads (mean 2.4 \pm 2.3 ) were sent from a single farm. To preserve the anonymity of the deer farmers, the cantons were grouped in six Swiss regions. The participation of deer farmers varied from 8 to $20 \%$ depending on the region (Fig. 1).

Of the 160 brains examined in this study, 40 were from diseased animals and 120 from healthy slaughtered deer. The age ranged between 2 and 17.5 years (mean $=6$ years for diseased animals and 10 years for slaughtered deer). Females represented $72 \%(n=28)$ of the diseased and $88 \%$ of the slaughtered animals. A complete necropsy could be performed on 28 diseased deer. Most of the investigated animals $(82.5 \%, n=132)$ were fallow deer (Dama dama), 9.4\% ( $n=15)$ European red deer (Cervus elaphus), 6.3\% $(n=10)$ sika deer (Cervus nippon) and $1.8 \%(n=3)$ elk.

\section{Laboratory investigations}

Four-micrometre-thick sections of paraffin-embedded tissue were stained with haematoxylin-eosin and exam-

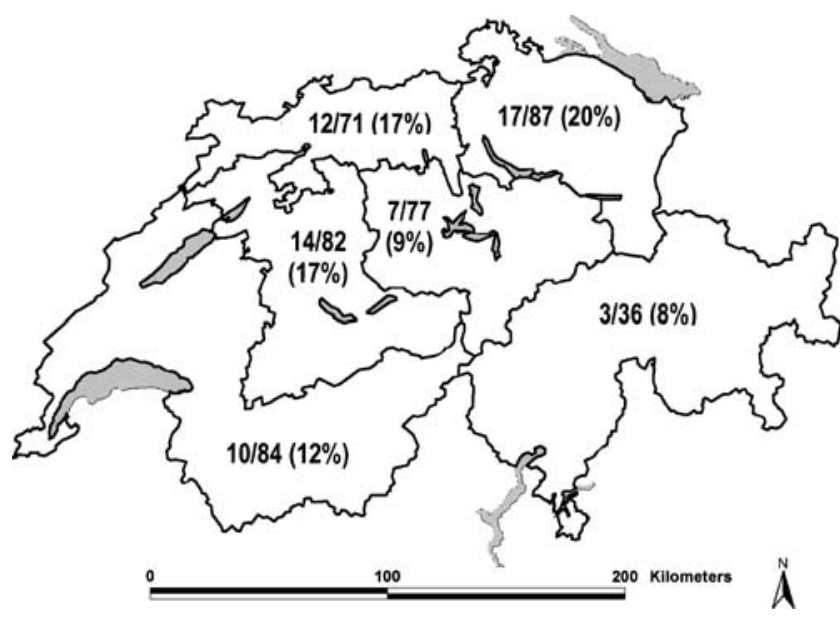

Fig. 1 Map of Switzerland (main lakes depicted in grey), divided into six geographical areas. The numbers $(X / Y)$ in each area indicate the total of deer farms that sent heads of dead animals for neurological analysis $(X)$ and the total of registered deer farms located in the area $(Y)$. The percentage of participation is indicated in parentheses

ined by light microscopy. Additionally, sections of the medulla oblongata at the level of the obex, lymph nodes and tonsils were stained by immunohistochemistry (IHC) to detect $\operatorname{PrP}^{\mathrm{CWD}}$. The monoclonal antibody F99/97.6.1 (VMRD, Pullman, WA) was used according to the manufacturer's suggestions. Slides were deparaffinised, and the tissue was pre-treated by denaturation in formic acid (98\%) for $30 \mathrm{~min}$, followed by autoclaving in citrate buffer at $121{ }^{\circ} \mathrm{C}$ for $20 \mathrm{~min}$. The sections were then incubated with F99/97.6.1 (at a dilution of 1:250) for $30 \mathrm{~min}$ at $37^{\circ} \mathrm{C}$. The visualisation was performed using a streptavidin-biotin kit (Dako ChemMate K5003, Dako, Denmark). As a positive control, we used sections of the brainstem of an American CWD case.

\section{Epidemiological calculations}

The evaluation of sample size for detection of disease in a population was performed according to Cannon and Roe (1982) with the computer program WinEpiscope 2.0. To estimate the maximal number of positive animals in the study population given that all samples showed negative results, we used the sample size (160 deer heads) and population size. In 2005, the Swiss captive deer population was estimated to be 9,400 animals, including roughly 5,200 deer older than 2 years (Sieber 2006).

\section{Results}

There was no macroscopic lesion in any of the brains, except for one case exhibiting suppurative meningitis. Histologically, $69 \%(n=110)$ of the brains were normal, whereas $6 \%(n=10)$ revealed changes that were classified as insignificant and 
were mostly old age changes, such as perivascular calcification or fibrosis of the plexus chorioideus. Lesions observed in the remaining $25 \%(n=40)$ of the deer were considered significant and divided into inflammatory $(3 \%, n=5)$ and degenerative lesions $(22 \%, n=35)$. Inflammatory changes were mild multifocal lymphocytic encephalitis and/or meningitis $(2 \%, n=3)$. One animal exhibited diffuse moderate suppurative meningitis, and another had a focal necrotizing meningoencephalitis (infection with Fusobacterium necrophorum). In 35 animals (22\%, 2 to 15 years old, slaughtered or diseased deer), mild to severe vacuolation of the white matter was observed (Fig. 2). Multifocal vacuolation was present in the optic tract, crus cerebri and thalamus or diffusely in the white matter. None of these animals showed any neurological symptoms.

None of the 160 examined animals, including 15 deer with clinical symptoms suggestive of CWD such as weight loss, progressive weakness, trembling and locomotional problems, exhibited any histological changes suspicious for CWD (Spraker et al. 2002a; Williams and Young 1993). IHC for $\mathrm{PrP}^{\mathrm{CWD}}$ was consistently negative, both in the obex region and in lymphoid tissues. A maximal possible CWD prevalence of 1.83 (95\% level of confidence [LC]) or $2.80 \%(99 \%$ LC) was estimated for the considered population.

\section{Discussion}

To our knowledge, this is the first published CWD survey based on histopathological examination of the whole brain in a European population of farmed deer. CWD could not be demonstrated in any of the 160 animals examined in this study. Detection of accumulated $\mathrm{PrP}^{\mathrm{CWD}}$ in the brain and lymphoid tissues is considered to be the method of choice to diagnose CWD (Miller and Williams 2002). The monoclonal antibody we applied (F99/97.6.1) is widely

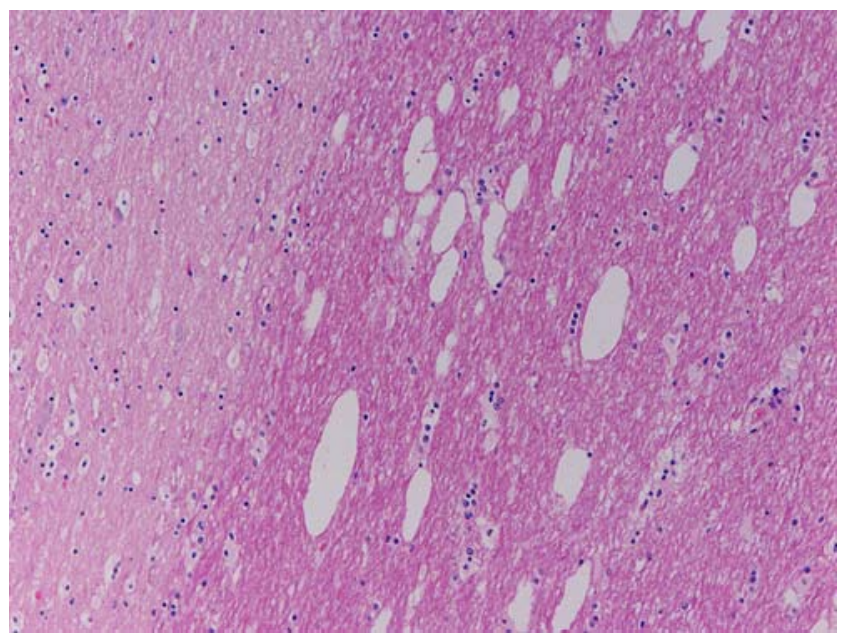

Fig. 2 Severe vacuolation in the optic tract of a symptomless 5-yearold deer (haematoxylin and eosin, 10×) used and has been validated for the mule deer in North America (Spraker et al. 2002b).

Composition of the study material was representative for the adult Swissfarmed deer population. Sample size should have been large enough for disease detection in case of an overall prevalence of greater than or equal to $1.8 \%(95 \%$ LC). Thus, this study does not definitively exclude the presence of CWD in the captive deer population in Switzerland but indicates that if it is present, prevalence is low. Reported CWD prevalences in infected herds or populations from North America are highly variable. Prevalences from less than $1 \%$ up to greater than $50 \%$ have been reported in infected captive herds (Kahn et al. 2004; Miller and Williams 2004; Williams and Miller 2002). In free-ranging wildlife, prevalence of preclinical CWD, based on histopathology and/or IHC, has been estimated at $1-15 \%$ within endemic areas (Williams and Miller 2002). The present study included mostly few deer from numerous distinct farms and was performed at animal level. Therefore, the obtained results allow the exclusion of a frequent CWD occurrence in the Swissfarmed deer population but not the infection of single herds.

For an expected overall prevalence of less than $1 \%$, more than 300 samples would have been necessary for disease detection. Although lots of efforts have been made to motivate deer breeders to participate in the study, this sample size could not be achieved, and a study at herd level was not realisable. Difficulties to obtain samples were probably due to the small herd sizes of Swiss deer farms (Sieber 2006), to the fact that it is not usual to slaughter adult deer and that participation was not compulsory.

The present survey comprised not only CWD but neurological diseases in general. It is interesting to note that $22 \%$ of the examined brains presented multifocal vacuolation of the white matter. This spongiosis was particularly severe in the optic tract. Spongy degeneration of the white matter can be seen in toxic or metabolic disorders, usually as a bilateral-symmetrical change (Summers et al. 1995a), and frequently in association with lesions in other organs. Whereas white matter vacuolation (spongiosis) is a common age-associated lesion reported in animals, it is usually associated with other histological changes, such as gliosis and accumulation of ceroid/ lipofuscin (Summers et al. 1995b), which were not observed in our cases. Incidental vacuolation of the white matter has been reported in more than $25 \%$ of examined brains from symptomless cattle (Gavier-Widen et al. 2001). As there was no correlation between the presence and degree of vacuolation and the age, symptoms observed (in particular, there was no report of a visual deficit) and other pathological changes in the examined deer in our study, the vacuolation ought to be considered as incidental. However, it would be interesting to pursue further investigations, 
mostly to exclude any occurrence of a sub-clinical intoxication in Swissfarmed deer.

We conclude that major neurological diseases, including CWD or other TSEs, are uncommon or possibly even absent in the Swissfarmed deer population. Nevertheless, it is advisable to further monitor the health status of captive cervids in Switzerland and to keep a high level of disease awareness among deer breeders and veterinarians.

Acknowledgements This survey was financed by the Swiss Federal Veterinary Office, with a contribution by the Swiss deer breeders association. Many thanks go to all deer breeders who made the study possible through their participation. We acknowledge the members of the committee of the Swiss deer breeders association for their valuable support during the planning and implementation of the project, as well as the technicians of the Institute of Animal Pathology and of the NeuroCenter, University of Berne. Particular thanks go to $H$. Segner for valuable support in the planning phase of the study, to J. Keller, H. -P. Dänzer and S. Murer for their active collaboration and to $\mathrm{M}$. Doherr for critical reading of the manuscript and epidemiological support. All performed experiments complied with the current laws of Switzerland.

\section{References}

Cannon RM, Roe RT (1982) Detecting the presence of disease. In: Cannon RM, Roe RT (eds) Livestock disease surveys, a field manual for veterinarians. Australian Government Publishing Service, Camberra, pp 14-15

De Bosschere H, Saegerman C, Neukermans A, Berkvens D, Casaer J, Vanopdenbosch E, Roels S (2006) First chronic wasting disease (CWD) surveillance of roe deer (Capreolus capreolus) in the northern part of Belgium. Vet Q 28:55-60

Gavier-Widen D, Wells GA, Simmons MM, Wilesmith JW, Ryan J (2001) Histological observations on the brains of symptomless 7year-old cattle. J Comp Pathol 124:52-59

Hamir AN, Miller JM, Cutlip RC, Kunkle RA, Jenny AL, Stack MJ, Chaplin MJ, Richt JA (2004) Transmission of sheep scrapie to elk (Cervus elaphus nelsoni) by intracerebral inoculation: final outcome of the experiment. J Vet Diagn Invest 16:316-321

Kahn S, Dube C, Bates L, Balachandran A (2004) Chronic wasting disease in Canada: Part 1. Can Vet J 45:397-404

Miller MW, Williams ES (2002) Detection of PrP(CWD) in mule deer by immunohistochemistry of lymphoid tissues. Vet Rec 151:610-612
Miller MW, Williams ES (2004) Chronic wasting disease of cervids. Curr Top Microbiol Immunol 284:193-214

Roels S, Saegermann C, De Bosschere H, Berkvens D, Gregoire F, Hoyoux A, Mousset B, Desmecht D, Vanopdenbosch E, Linden A (2005) First results of chronic wasting disease (CWD) surveillance in the South-Eastern part of Belgium. Vet Q 27:98-104

Schettler E, Steinbach F, Eschenbacher-Kaps I, Gerst K, Meussdoerffer F, Risch K, Streich WJ, Froelich K (2006) Surveillance for prion disease in cervids, Germany. Emerg Infect Dis 12:319-322

Scientific Steering Committee (2003) Chronic wasting disease and tissues that might carry a risk for human food and animal feeding chains. In: European Commission, Scientific Steering Committee. Available at: http://europa.eu.int $/ \mathrm{comm} / \mathrm{food} / \mathrm{fs} / \mathrm{sc} / \mathrm{ssc} /$ outcome_en.html\#reports. Accessed 13 June 2005

Sieber V (2006) Dissertation, Veterinary Medicine. University of Berne, Berne

Sohn HJ, Kim JH, Choi KS, Nah JJ, Joo YS, Jean YH, Ahn SW, Kim OK, Kim DY, Balachandran A (2002) A case of chronic wasting disease in an elk imported to Korea from Canada. J Vet Med Sci 64:855-858

Spraker TR, O'Rourke KI, Balachandran A, Zink RR, Cummings BA, Miller MW, Powers BE (2002a) Validation of monoclonal antibody F99/97.6.1 for immunohistochemical staining of brain and tonsil in mule deer (Odocoileus hemionus) with chronic wasting disease. J Vet Diagn Invest 14:3-7

Spraker TR, Zink RR, Cummings BA, Wild MA, Miller MW, O'Rourke KI (2002b) Comparison of histological lesions and immunohistochemical staining of proteinase-resistant prion protein in a naturally occurring spongiform encephalopathy of free-ranging mule deer (Odocoileus hemionus) with those of chronic wasting disease of captive mule deer. Vet Pathol 39:110-119

Summers BA, Cummings JF, de Lahunta A (1995a) Degenerative diseases of the central nervous system. In: Summers BA, Cummings JF, de Lahunta A (eds) Veterinary neuropathology. Mosby-Year Book, St. Louis, MO, pp 208-350

Summers BA, Cummings JF, de Lahunta A (1995b) Neuropathology of aging. In: Summers BA, Cummings JF, de Lahunta A (eds) Veterinary neuropathology. Mosby-Year Book, St. Louis, MO, pp 49-55

Williams ES, Young S (1993) Neuropathology of chronic wasting disease of mule deer (Odocoileus hemionus) and elk (Cervus elaphus nelsoni). Vet Pathol 30:36-45

Williams ES, Miller MW (2002) Chronic wasting disease in deer and elk in North America. Rev Sci Tech 21:305-316

Williams ES, Miller MW (2003) Transmissible spongiform encephalopathies in non-domestic animals: origin, transmission and risk factors. Rev Sci Tech 22:145-156 